\title{
Integral Apollonian Packings
}

\section{Peter Sarnak}

Abstract. We review the construction of integral Apollonian circle packings. There are a number of Diophantine problems that arise in the context of such packings. We discuss some of them and describe some recent advances.

1. AN INTEGRAL PACKING. The quarter, nickel, and dime in Figure 1 are placed so that they are mutually tangent. This configuration is unique up to rigid motions. As far as I can tell there is no official exact size for these coins, but the diameters of 24, 21 ,

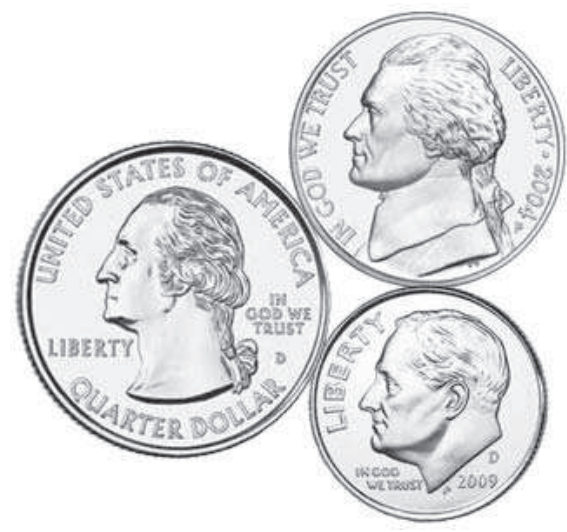

Figure 1.

and 18 millimeters are accurate to the nearest millimeter and I assume henceforth that these are the actual diameters. Let $C$ be the unique (see below) circle that is tangent to the three coins as shown in Figure 2. It is a small coincidence that its diameter is rational, as indicated.

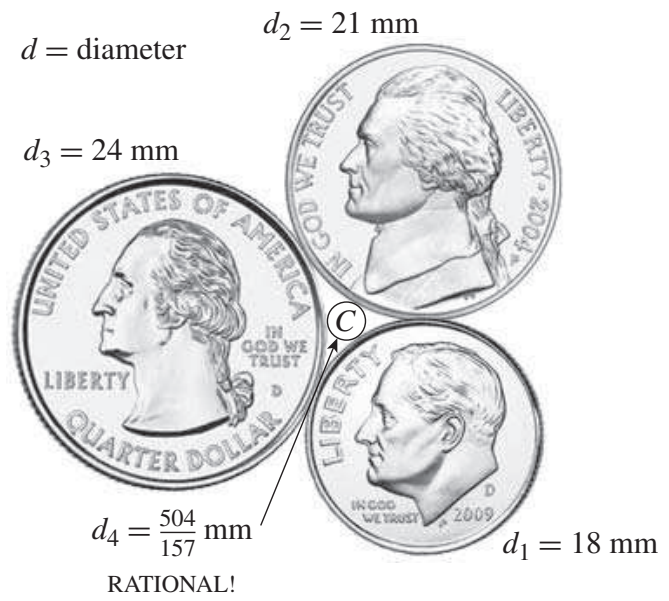

Figure 2.

doi:10.4169/amer.math.monthly.118.04.291 
What is more remarkable is that if we continue to place circles in the resulting regions bounded by three circles as described next, then all the diameters are rational. Since the circles become very small, so do their radii, and it is more convenient to work with their curvatures, which are the reciprocals of the radii. In fact in this example it is natural to scale everything further by 252 , so let us take $252 \mathrm{~mm}$ as our unit of measurement, and then for each circle $C$ let $a(C)$ be the curvature of $C$ in these units. With this rescaling, all of the curvatures turn out to be integers.

In Figure 3 our three tangent circles are displayed together with the unique outer mutually tangent circle. The $a(C)$ for each circle is depicted inside the circle. Note that the outer circle has a negative sign indicating that the other circles are in its interior (it is the only circle with a negative sign).

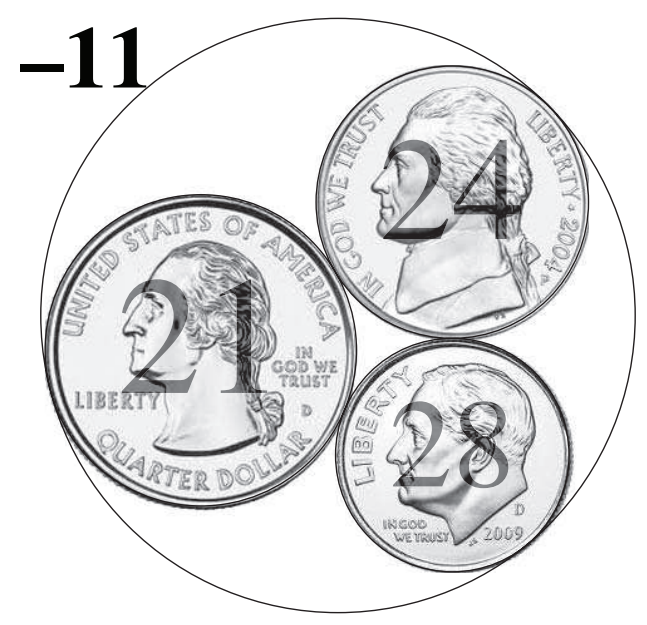

Figure 3.

At the next generation we place circles in each of the 4 lune regions, obtaining the configuration in Figure 4 with the curvatures $a(C)$ as indicated. For the 3rd generation we will fill in the 12 new lunes as in Figure 5. Continuing in this way ad infinitum yields the integral Apollonian packing $P_{0}$ depicted in Figure 6.

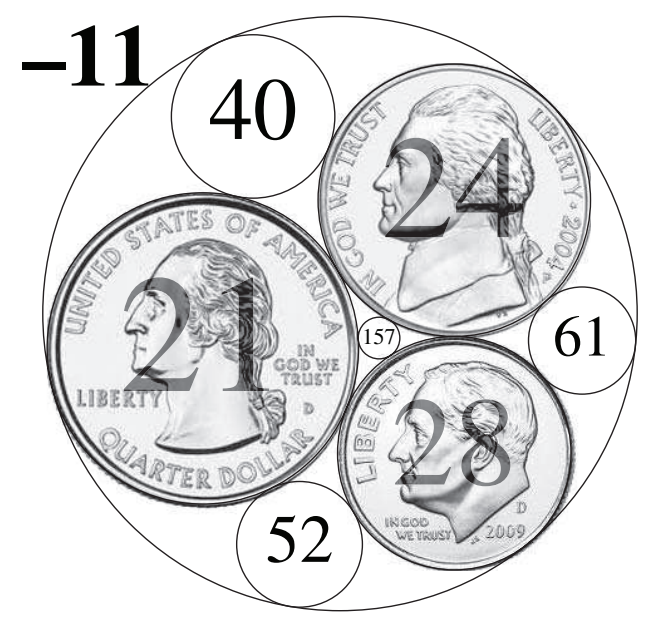

Figure 4. 


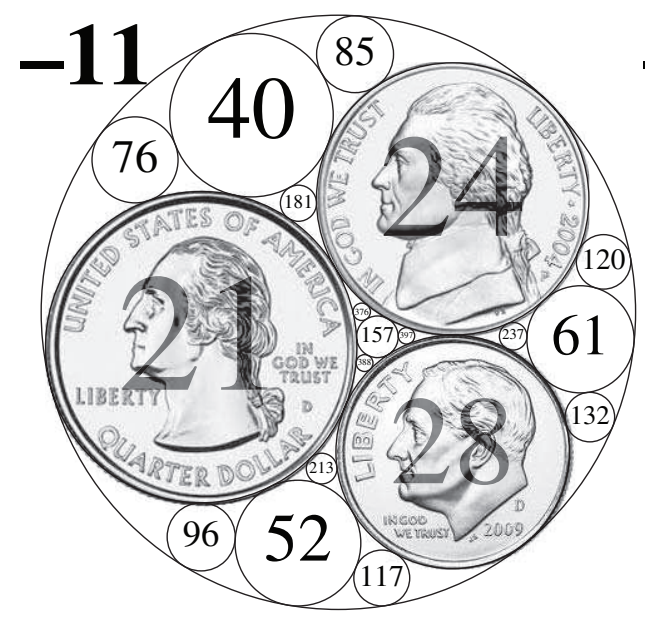

Figure 5.

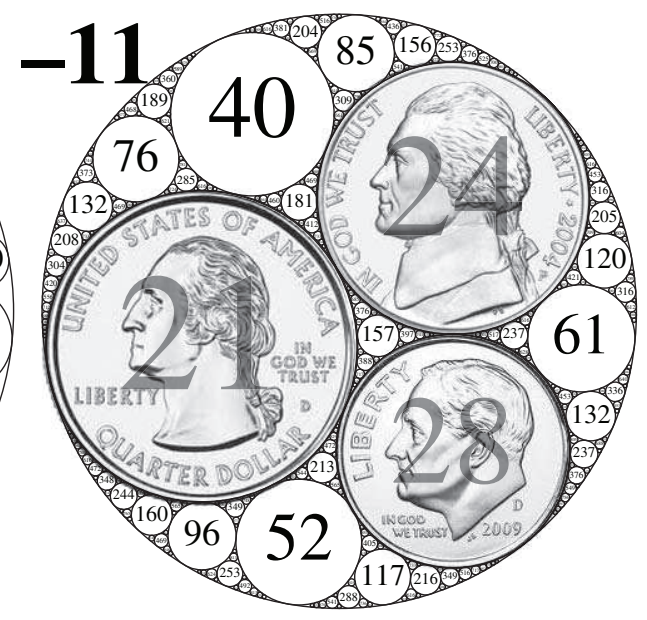

Figure 6.

My aim in this paper is first to explain the elementary plane geometry behind the above construction, and then to discuss the Diophantine properties of the integers appearing as curvatures in integral Apollonian packings such as $P_{0}$. As with many problems in number theory, the basic questions here are easy to state but difficult to resolve. There are many papers in the literature dealing with Apollonian packings and their generalizations. However, the Diophantine questions are quite recent and are raised in the lovely five-author paper [10]. The developments that we discuss below are contained in the letter and preprints [19], [14], [8], [9], [2], and [1].

In Section 2 we review (with proofs) some theorems from Euclidean geometry that are central to understanding the construction of $P_{0}$. This requires no more than high school math. The proofs of the results in later sections involve some advanced concepts and so we only outline these proofs in general terms. However, the notions involved in the statements of all the theorems are ones that are covered in basic undergraduate courses, and it is my hope that someone with this background can follow the discussion to the end. In Section 3 we introduce the key object $A$, which is the symmetry group of $P_{0}$. It is a group of $4 \times 4$ integer matrices that is deficient in a way that makes its study both interesting and challenging. Section 4 deals with the basic analytic question of counting the number of circles in $P_{0}$ when they are ordered by their curvatures. Sections 5 and 6 are concerned with Diophantine questions such as which numbers are curvatures of circles in $P_{0}$, a possible local-to-global principle, and the number of circles whose curvatures are prime numbers.

2. APOLLONIUS'S AND DESCARTES' THEOREMS. First some notation. $P$ denotes an integral Apollonian packing and $C$ a typical circle in $P$. Denote by $r(C)$ its radius and by $a(C)=1 / r(C)$ its curvature. Let $w(C)$ be the generation $n \geq 1$ at which $C$ first appears in the packing. Thus for $n>1$, there are $4 \cdot 3^{n-2}$ new circles that are placed at the $n$th generation.

Apollonius's Theorem. Given three mutually tangent circles $C_{1}, C_{2}, C_{3}$, there are exactly two circles $C$ and $C^{\prime}$ tangent to all three.

Our proof is based on the use of motions of the plane that take circles to circles (we allow a straight line as a circle with "infinite" radius) and preserve tangencies and angles. Specifically, the operation of inversion in a circle $E$ of radius $r$ and center $O$ as 
displayed in Figure 7 is such an operation. The transformation takes $p$ to $q$ as shown, and one checks that it satisfies the above properties.

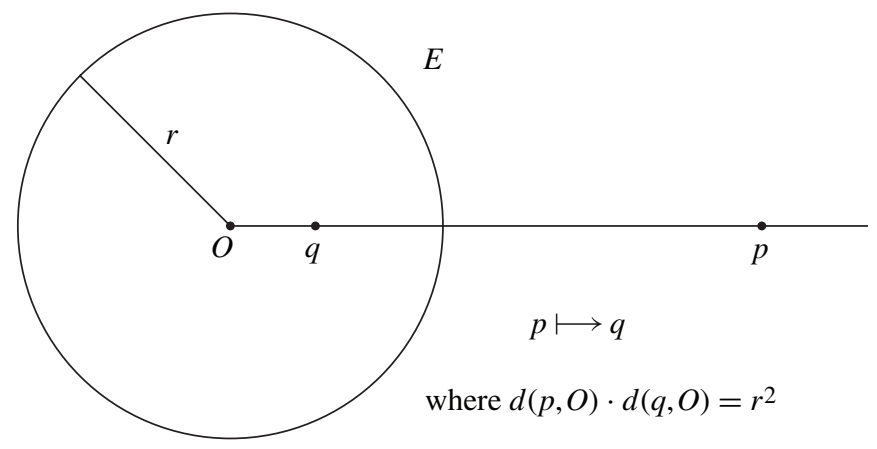

Figure 7.

To prove Apollonius's theorem, let $C_{1}, C_{2}, C_{3}$ be as shown in Figure 8 and let $\xi$ be the point of tangency between $C_{1}$ and $C_{2}$.

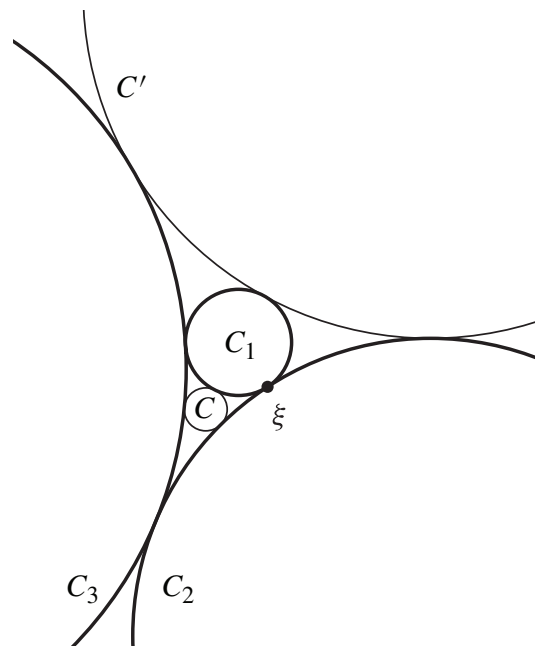

Figure 8.

Invert Figure 8 in a circle $E$ centered at $\xi$. Then $C_{1}$ and $C_{2}$ are mapped to circles through infinity, that is, parallel straight lines $\tilde{C}_{1}$ and $\tilde{C}_{2}$, while $C_{3}$ is mapped to a circle $\tilde{C}_{3}$ tangent to both as indicated in Figure 9.

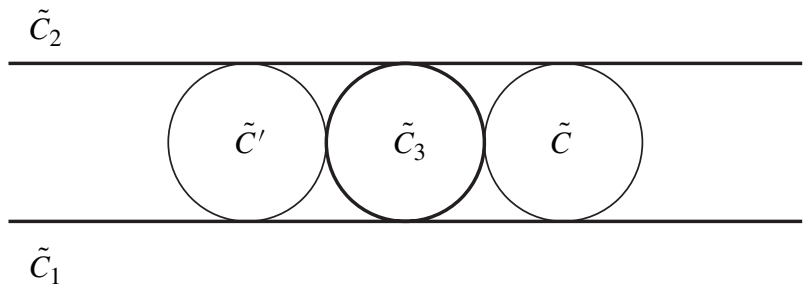

Figure 9. 
In this configuration, where $\tilde{C}_{1}$ and $\tilde{C}_{2}$ are parallel lines, it is clear that there are exactly two circles, $\tilde{C}^{\prime}$ and $\tilde{C}$, which are mutually tangent to $\tilde{C}_{1}, \tilde{C}_{2}$, and $\tilde{C}_{3}$. Hence by inverting again, Apollonius's theorem follows.

Descartes' Theorem. Given four mutually tangent circles whose curvatures are $a_{1}, a_{2}, a_{3}, a_{4}$ (with our sign convention), then

$$
F\left(a_{1}, a_{2}, a_{3}, a_{4}\right)=0,
$$

where $F$ is the quadratic form

$$
F(a)=2 a_{1}^{2}+2 a_{2}^{2}+2 a_{3}^{2}+2 a_{4}^{2}-\left(a_{1}+a_{2}+a_{3}+a_{4}\right)^{2} .
$$

Proof. Again we employ inversion.

We need a couple of formulae relating the radius of a circle and its inversion in $E$ of radius $k$.

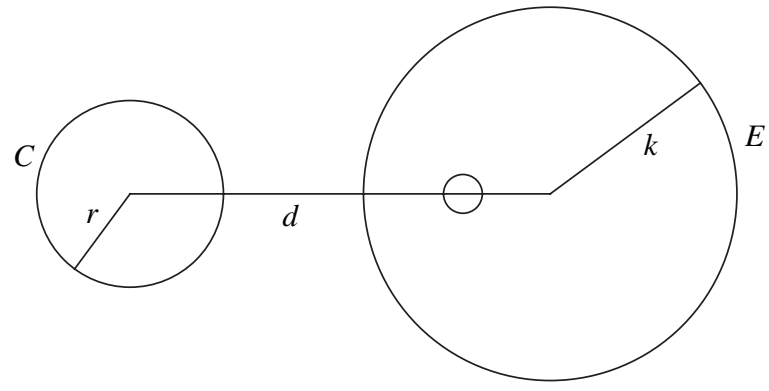

Figure 10.

Inverting $C$ in $E$ yields a circle of radius

$$
k^{2} r /\left(d^{2}-r^{2}\right),
$$

where $r$ is the radius of $C$ and $d$ the distance between the centers of $C$ and $E$.

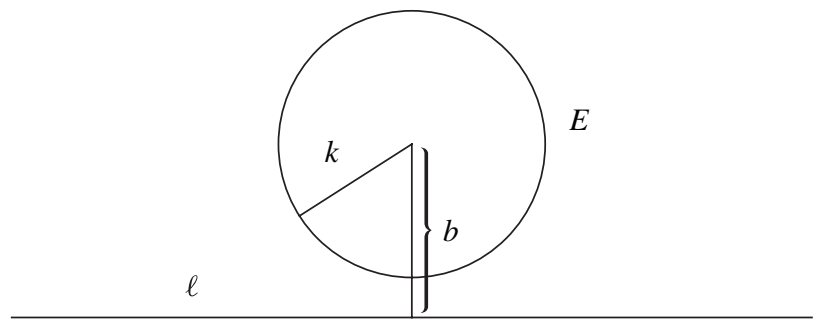

Figure 11.

Inverting the straight line $\ell$ in $E$ yields a circle of radius

$$
k^{2} / 2 b,
$$

where $b$ is the distance from the center of $E$ to $\ell$. 


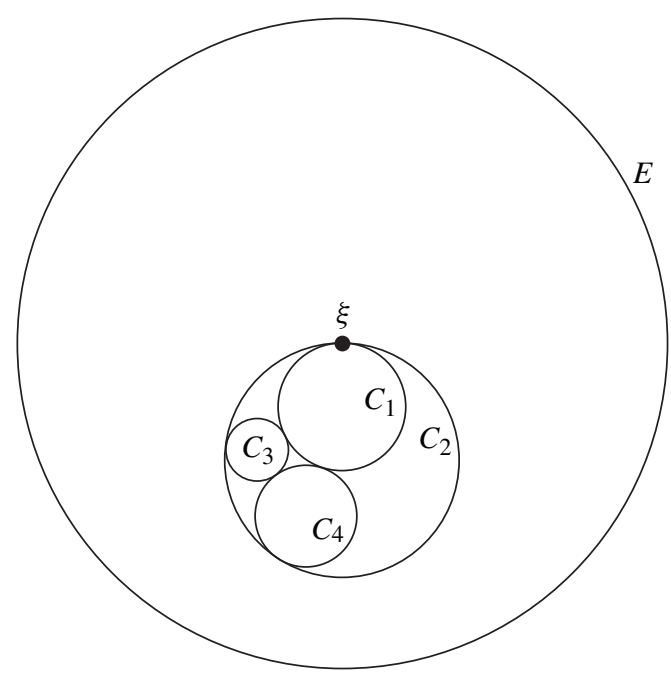

INVERT IN $E$

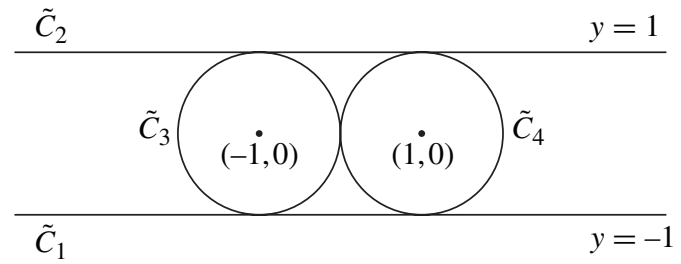

Figure 12.

Now let $C_{1}, C_{2}, C_{3}, C_{4}$ be our four mutually tangent circles as shown in Figure 12. Let $E$ be a circle centered at $\xi=\left(x_{0}, y_{0}\right)$, the point of tangency of $C_{1}$ and $C_{2}$. Inverting in $E$ we arrive at the configuration $\tilde{C}_{1}, \tilde{C}_{2}, \tilde{C}_{3}, \tilde{C}_{4}$ as shown (after further translation and rotation and assuming $\left.y_{0}>1\right)$.

Applying (1) and (2) above we find that

$$
\begin{array}{ll}
r\left(C_{3}\right)=\frac{k^{2}}{x_{0}^{2}-2 x_{0}+y_{0}^{2}}, & r\left(C_{4}\right)=\frac{k^{2}}{x_{0}^{2}+2 x_{0}+y_{0}^{2}} \\
r\left(C_{2}\right)=\frac{k^{2}}{2\left(y_{0}-1\right)}, & r\left(C_{1}\right)=\frac{k^{2}}{2\left(y_{0}+1\right)},
\end{array}
$$

where $k$ is the radius of $E$. Substituting $a\left(C_{i}\right)=1 / r\left(C_{i}\right)$ for $i=1,3$, and 4 and $a\left(C_{2}\right)=-1 / r\left(C_{2}\right)$, according to our sign convention, in the Descartes form $F$ and doing some algebraic manipulation yields $F\left(a_{1}, a_{2}, a_{3}, a_{4}\right)=0$ (see Coxeter [6] for a further discussion). This proof of Descartes' theorem is a little unsatisfying in that it requires some calculation at the end, but it is conceptually simple. That is, the proof is no more than inversion and keeping track of the quantities under this transformation.

We can now complete our discussion of the packing $P_{0}$ or any other integral packing. First, according to Apollonius's theorem the placement of each circle in each lune region is unique once we have a starting configuration of three mutually tangent circles $C_{1}, C_{2}, C_{3}$. Thus these circles determine the entire packing. Now suppose that these 
starting circles have curvatures $a_{1}, a_{2}, a_{3}$. Then, according to Descartes' theorem, if $C$ and $C^{\prime}$ are the two circles tangent to $C_{1}, C_{2}, C_{3}$, then their curvatures $a_{4}$ and $a_{4}^{\prime}$ satisfy

$$
\left.\begin{array}{l}
F\left(a_{1}, a_{2}, a_{3}, a_{4}\right)=0, \\
F\left(a_{1}, a_{2}, a_{3}, a_{4}^{\prime}\right)=0 .
\end{array}\right\}
$$

Thus $a_{4}$ and $a_{4}^{\prime}$ are roots of the same quadratic equation, and using the quadratic formula one finds that

$$
\begin{aligned}
a_{4}+a_{4}^{\prime} & =2 a_{1}+2 a_{2}+2 a_{3}, \\
a_{4}, a_{4}^{\prime} & =a_{1}+a_{2}+a_{3} \pm 2 \sqrt{\triangle},
\end{aligned}
$$

where

$$
\triangle=a_{1} a_{2}+a_{1} a_{3}+a_{2} a_{3}
$$

So for our three coins with curvatures 21, 24, and 28 in Figure 3 we have that $\triangle=$ $1764=(42)^{2}$. Hence $a_{4}$ and $a_{4}^{\prime}$ are integers, and this is the small coincidence that leads to $P_{0}$ being an integral packing. Indeed for a general packing with starting curvatures $a_{1}, a_{2}, a_{3}$, the curvatures $a_{4}$ and $a_{4}^{\prime}$ are expressed in terms of $a_{1}, a_{2}, a_{3}$, and $\sqrt{\triangle}$. Now starting with these four circles, $C_{1}, C_{2}, C_{3}, C_{4}$, we get all future circles in the packing by taking three circles at a time and using the existing fourth mutually tangent circle to produce another such in the packing. In doing so we don't need to extract any further square roots. Thus the curvatures of the entire packing $P$ are expressed as sums of the quantities $a_{1}, a_{2}, a_{3}, \sqrt{\triangle}$, with integer coefficients. In particular, when $a_{1}, a_{2}, a_{3}$ are integers and $\Delta$ is a perfect square, the packing is an integral packing. In terms of the radii $r_{1}, r_{2}, r_{3}$, which we assume are rational numbers, the further radii of circles in the packing lie in the field of rationals with $\sqrt{\left(r_{1}+r_{2}+r_{3}\right) r_{1} r_{2} r_{3}}$ adjoined.

3. THE APOLLONIAN GROUP. A deeper study of an Apollonian packing is facilitated by introducing the symmetry group $A$, which is called the Apollonian group. Given 4 mutually tangent circles in a packing whose curvatures are $\left(a_{1}, a_{2}, a_{3}, a_{4}\right) \in$ $\mathbb{R}^{4}$ we get 4 new such configurations by taking the 4 subsets of 3 of the 4 original circles and in each case introducing a new circle in the packing using Apollonius's theorem. So if $C_{1}, C_{2}, C_{3}, C_{4}$ is our starting configuration and we take the subset $C_{1}, C_{2}, C_{3}$ and generate $C_{4}^{\prime}$ from $C_{4}$, we get a new configuration $C_{1}, C_{2}, C_{3}, C_{4}^{\prime}$ in the packing, with $C_{4}^{\prime}$ being the new circle in the corresponding lune region. According to (4) the new 4-tuple of curvatures is $a^{\prime}=\left(a_{1}, a_{2}, a_{3}, a_{4}^{\prime}\right)$, where in matrix notation

$$
a^{\prime}=a S_{4}
$$

and

$$
S_{4}=\left[\begin{array}{rrrr}
1 & 0 & 0 & 2 \\
0 & 1 & 0 & 2 \\
0 & 0 & 1 & 2 \\
0 & 0 & 0 & -1
\end{array}\right]
$$

Taking the other subsets of $C_{1}, C_{2}, C_{3}, C_{4}$ yields

$$
a^{\prime}=a S_{j}, \quad j=1,2,3,
$$


with

$$
S_{1}=\left[\begin{array}{rrrr}
-1 & 0 & 0 & 0 \\
2 & 1 & 0 & 0 \\
2 & 0 & 1 & 0 \\
2 & 0 & 0 & 1
\end{array}\right], \quad S_{2}=\left[\begin{array}{rrrr}
1 & 2 & 0 & 0 \\
0 & -1 & 0 & 0 \\
0 & 2 & 1 & 0 \\
0 & 2 & 0 & 1
\end{array}\right], \quad S_{3}=\left[\begin{array}{rrrr}
1 & 0 & 2 & 0 \\
0 & 1 & 2 & 0 \\
0 & 0 & -1 & 0 \\
0 & 0 & 2 & 1
\end{array}\right] .
$$

Note that the $S_{j}$ have integer entries and that

$$
S_{j}^{2}=I
$$

Definition. The Apollonian group $A$ is the subgroup of the $4 \times 4$ integer matrices of determinant $\pm 1\left(\mathrm{GL}_{4}(\mathbb{Z})\right)$ generated by $S_{1}, S_{2}, S_{3}, S_{4}$.

The transformations $S_{j}$, as well as those generated by them, switch the roots of one coordinate as in equation (3). So the group $A$ arises from Galois symmetries and it also acts as a symmetry of the packing. Indeed, according to our discussion above the 4-tuples of curvatures of mutually tangent circles in a packing $P$ are the orbits $\mathcal{O}_{a}=a \cdot A$ of $A$, where $a$ is any such tuple in the packing. If $a$ is integral then so is any $x$ in $\mathcal{O}_{a}$, and if $a$ is primitive (that is, its coordinates have no common factor) then so is every $x \in \mathcal{O}_{a}$.

We assume that our packing $P$, like $P_{0}$, is integral, primitive, and bounded (as in Figure 6). In this case any $x \in \mathcal{O}_{a}$ is a primitive integral point, which by Descartes' theorem lies on the cone $V$ given by

$$
V=\{x: F(x)=0\} .
$$

It is clear, and one can check it directly, that

$$
F\left(x S_{j}\right)=F(x) \quad \text { for } x \in \mathbb{R}^{4} \text { and } j=1,2,3,4 .
$$

Hence

$$
F(x \gamma)=F(x) \text { for any } x \text { and } \gamma \in A \text {. }
$$

Let $O_{F}$ be the orthogonal group of $F$, that is,

$$
O_{F}=\left\{g \in \mathrm{GL}_{4}: F(x g)=F(x)\right\} .
$$

$O_{F}$ is an "algebraic group" in that it is defined by algebraic (in this instance quadratic) equations in $\left(x_{i j}\right), i, j=1,2,3,4$. Explicitly it is given by:

$$
X S X^{t}=S,
$$

where $S$ is the matrix of $F$, that is,

$$
F(x)=x S x^{t} .
$$

From (12) we have that

$$
A \leq O_{F}(\mathbb{Z})
$$

where $O_{F}(\mathbb{Z})$ consists of the matrices in $O_{F}$ whose entries are integers. 
This brings us to the heart of the matter, at least as far as Diophantine properties of an integral packing are concerned. The group $O_{F}(\mathbb{Z})$ is a much-studied and wellunderstood group. It is an "arithmetic" group and as such is central in the arithmetic theory of quadratic forms (for example in connection with understanding which integers are represented by an integral quadratic form) and also in automorphic forms. It is also big, as is demonstrated by the orbit of a primitive integral point $x \in V^{\text {prim }}(\mathbb{Z})$ under $O_{F}(\mathbb{Z})$ being all of $V^{\text {prim }}(\mathbb{Z})$. The salient features of the Apollonian group $A$ are:

(i) $A$ is small; it is of infinite index in $O_{F}(\mathbb{Z})$.

(ii) $A$ is not too small; it is Zariski dense in $O_{F}$.

Statement (i) makes the Diophantine analysis of an integral packing nonstandard in that the familiar arithmetic tools don't apply. Statement (ii) says that $A$ is large in the algebraic geometric sense that any polynomial in the variables $x_{i j}(i, j=1,2,3,4)$ of $4 \times 4$ matrices that vanishes on $A$ must also vanish on the complex points of $O_{F}$. It is a modest condition on $A$ and it plays a critical role in understanding what $A$ looks like when reduced in arithmetic modulo $q$, for $q>1$.

An instructive of way of seeing (i) is to consider the orbits of $A$ on $V^{\text {prim }}(\mathbb{Z})$. These correspond to the different integral primitive Apollonian packings, and there are infinitely many of them. In [10] it is shown how to use $A$ to find a point $v$ in each orbit $a A$ called a "root quadruple" which is a reduced element, the definition of reduced being that $v=\left(a_{1}, a_{2}, a_{3}, a_{4}\right)$ is in $V^{\text {prim }}(\mathbb{Z})$ and satisfies $a_{1}+a_{2}+a_{3}+a_{4}>0$, $a_{1} \leq 0 \leq a_{2} \leq a_{3} \leq a_{4}$, and $a_{1}+a_{2}+a_{3} \geq a_{4}$. For example, for $P_{0}$, the reduced $v$ is $(-11,21,24,28)$. There are infinitely many root quadruples; in fact, one can count their number asymptotically when they are ordered by the Euclidean norm (see [18]).

4. COUNTING CIRCLES IN A PACKING. In order to investigate the Diophantine properties of a packing $P$ we need to count the circles in $P$. There are at least two useful ways to order the circles:

$(\alpha)$ By the size of the curvature. Let

$$
N_{P}(x):=|\{C \in P: a(C) \leq x\}| .
$$

$(\beta)$ Combinatorially by the generation $w(C)$. There are $4 \cdot 3^{n-2}$ circles at generation $n$; what is their typical curvature?

The answers to these lie in noncommutative harmonic analysis. As to the first, let $\delta(P)$ be the exponent of convergence of the series

$$
\sum_{C \in P} r(C)^{s}
$$

That is, for $s>\delta$ the series converges, while for $s<\delta$ it diverges. Clearly $\delta$ is at most 2 since $\pi \sum_{C \in P} r(C)^{2}$ is finite (it is twice the area of the circle enclosing $P$ ). On the other hand $\sum_{C \in P} r(C)$ is infinite (see [20] for an elegant proof), and hence $1 \leq \delta(P) \leq 2$. Also $\delta$ doesn't depend on $P$, since any two packings are equivalent by a Möbius transformation, that is, a motion of the complex plane $\mathbb{C}$ by a conformal (angle preserving) transformation $z \rightarrow(\alpha z+\beta)(\gamma z+\eta)^{-1}, \alpha \eta-\beta \gamma=1$. So $\delta=$ $\delta(A)$ is an invariant of the Apollonian group and it is known to have many equivalent definitions. It can be estimated: [17] gives $\delta=1.30568 \ldots$. Using elementary methods 
Boyd [3] shows that

$$
\lim _{x \rightarrow \infty} \frac{\log N_{P}(x)}{\log x}=\delta .
$$

Very recently Kontorovich and Oh [14] have determined the asymptotics for $N_{P}(x)$. Their method uses ergodic properties of flows on $A \backslash O_{F}(\mathbb{R})$ and in particular the LaxPhillips spectral theory for the Laplacian on the infinite-volume hyperbolic three manifold $X=A \backslash O_{F}(\mathbb{R}) / K$, where $K$ is a maximal compact subgroup of $O_{F}(\mathbb{R})$, as well as the Patterson-Sullivan theory for the base eigenfunction on $X$.

Theorem [14]. There is a positive $b=b(P)$ such that

$$
N_{P}(x) \sim b x^{\delta}, \quad \text { as } x \rightarrow \infty .
$$

Numerical calculations [9] indicate that $b\left(P_{0}\right)=0.0458 \ldots$

As far as $(\beta)$ goes, Furstenberg's theory of random products of matrices, in this case $S_{j 1} S_{j 2} \cdots S_{j m}$ with $j_{k} \in\{1,2,3,4\}$ and $j_{k} \neq j_{k+1}$ for any $k$, and in particular the positivity of the Lyapunov exponent $\gamma$ associated with such products, dictates the distribution of the numbers $\log a(C)$ with $w(C)=m$. In fact there is a central limit theorem [15], which asserts that this distribution has mean $\gamma m$ and variance of size $\sqrt{m}$ as $m$ tends to infinity. Here $\gamma=\gamma(A) \cong 0.9149 \ldots$ according to Fuchs [8], who has done some numerical simulation.

5. DIOPHANTINE ANALYSIS. Which integers occur as curvatures of circles $C$ in an integral packing $P$ ? According to the theorem in the last section, the number of $a(C)$ 's less than $x$ with $C \in P$ (counted with multiplicities) is about $x^{\delta}$, and hence one might expect that a positive proportion of all numbers occur as curvatures. This was conjectured in [10]. An approach to this conjecture using the subgroups $B_{1}, B_{2}, B_{3}, B_{4}$, with $B_{1}=\left\langle S_{2}, S_{3}, S_{4}\right\rangle$, etc., was introduced in [19]. The point is that unlike $A, B_{j}$ is an arithmetic subgroup of $H_{j}(\mathbb{R})$, where $H_{j}$ is the Zariski closure of $B_{j}$. In this way the study of the integer orbits of $B_{j}$ falls under the realm of the arithmetic theory of quadratic forms. In particular, one finds that among the curvatures are the values at integers of various inhomogeneous binary quadratic forms. Very recently Bourgain and Fuchs have shown that the different forms are highly uncorrelated at certain scales, and as a consequence they establish:

Theorem [1]. The positive density conjecture is true, that is, the set of curvatures in an integral Apollonian packing has positive density in all the positive integers.

A much more ambitious conjecture about the set of numbers which are curvatures is that it should satisfy a local-to-global principle. According to the asymptotics for $N_{P}(x)$, we have that, on average, a large integer $n$ is hit about $n^{\delta-1}$ times. So if $n$ is large, one might hope that $n$ is in fact hit unless there is some obvious reason that it shouldn't be. The obvious reason is that the $n$ 's that are curvatures satisfy congruence conditions and these can be studied in detail.

It is here that $A$ being Zariski dense in $O_{F}$ is relevant. There are general theorems [16] which assert that for groups such as $A$ and $q$ a positive integer having its prime factors outside a finite set of primes $S=S(A)$, the reduction of $A \bmod q$ in $\mathrm{GL}_{4}(\mathbb{Z} / q \mathbb{Z})$ is the same as that of $O_{F}(\mathbb{Z}) \bmod q$. While the description of the last is still a bit complicated because orthogonal groups don't quite satisfy strong approximation (see [4]; 
one needs to pass to the spin double cover), it is nevertheless well understood. For the Apollonian group $A$, Fuchs [8] has determined the precise image of $A$ in $\mathrm{GL}_{4}(\mathbb{Z} / q \mathbb{Z})$ for every $q$. In particular the "ramified" set $S(A)$ consists of only 2 and 3 .

From her characterization one obtains the following important product structure for the reduced orbits [8]. Let $\mathcal{O}_{a}(q)$ be the reduction of $\mathcal{O}_{a}$ into $(\mathbb{Z} / q \mathbb{Z})^{4}$. If $q=q_{1} q_{2}$ with $\left(q_{1}, q_{2}\right)=1$, then $\mathcal{O}_{a}(q)=\mathcal{O}_{a}\left(q_{1}\right) \times \mathcal{O}_{a}\left(q_{2}\right)$ as subsets of $(\mathbb{Z} / q \mathbb{Z})^{4}=$ $\left(\mathbb{Z} / q_{1} \mathbb{Z}\right)^{4} \times\left(\mathbb{Z} / q_{2} \mathbb{Z}\right)^{4}$ (the latter identification coming from the Chinese remainder theorem). Moreover for $p \geq 5$ a prime and $e \geq 1, \mathcal{O}_{a}\left(p^{e}\right)=V\left(\mathbb{Z} / p^{e} \mathbb{Z}\right) \backslash\{0\}$, that is, the nonzero points on the Descartes cone in arithmetic $\bmod p^{e}$. For $p=2$ and 3 this is not true, but the description of $\mathcal{O}_{a}\left(p^{e}\right)$ stabilizes at $e=3$ for $p=2$ and at $e=1$ for $p=3$. With this detailed information about the orbits $\bmod q$ it is a simple matter to determine the exact congruence conditions that the curvatures in an integral packing must satisfy.

For example for the packing $P_{0}$, the reader might have noticed that

$$
a(C) \equiv 0,4,12,13,16,21(\bmod 24)
$$

and this is the only congruence restriction. The local-to-global conjecture for $P_{0}$ (and a similar conjecture applies to any integral $P$ ) is then:

Local-to-Global Conjecture ([10], [9]). Except for finitely many $m \geq 1$, every $m$ satisfying (17) is the curvature of some $C \in P_{0}$.

If the conjecture is true and is proven effectively, then one would have a completely satisfactory description of the set of curvatures. Fuchs and Sanden [9] have made a detailed numerical study of this local-to-global conjecture. For $P_{0}$ they list the $N_{P_{0}}\left(10^{8}\right)$ circles with curvatures at most $10^{8}$ and they examine those with $10^{7} \leq a(C)<10^{8}$, grouping them into each of the six allowed progressions mod 24. For each progression the distribution of the frequencies with which the numbers are hit is calculated. The means of these distributions can be determined asymptotically using [8] and [14], and it is smallest for $m \equiv 0(\bmod 24)$ and largest for $m \equiv 21(\bmod 24)$; in fact the latter is double the former. The results of these calculations for these two progressions are displayed by the histograms in Figures 13 and 14. The number of exceptions, that is, numbers in $\left[10^{7}, 10^{8}\right.$ ) which satisfy the congruence but are not curvatures (which is the frequency of 0 in the histogram), is still sizable for $m \equiv 0(\bmod 24)$, while for $m \equiv 21$ $(\bmod 24)$ it is the single number 11459805 . The reason for the difference is that the mean for $m \equiv 0(\bmod 24)$ is still quite small at 12.41 , while for $m \equiv 21(\bmod 24)$ it is 24.86. As $x$ increases, the mean in each progression will be of order $x^{\delta-1}$ and the frequency of 0 will drop. The local-to-global principle asserts that from some point on this frequency count doesn't change, and it appears to be quite plausible. From this data one might reasonably venture that every $m \equiv 21(\bmod 24)$ bigger than 11459805 is a curvature of a circle in $P_{0}$.

To put this local-to-global conjecture in perspective, consider the same problem for $O_{F}(\mathbb{Z})$, rather than for $A$. That is, consider the question of which numbers $a$ are coordinates of points $x \in V^{\text {prim }}(\mathbb{Z})$. For a given $a$ this is a question of representing an integer by a ternary quadratic form. For the form at hand this is not a difficult problem (every $a$ occurs), but if one were to change the form $F$, the resulting form in three variables would be quite general. That is, one is facing the question of a localto-global principle (except for finitely many exceptions) for ternary quadratic forms. This is the most difficult case of Hilbert's 11th problem, and it has only recently been settled in general (see [7], [5]). Even there the solution is ineffective and the local-to- 


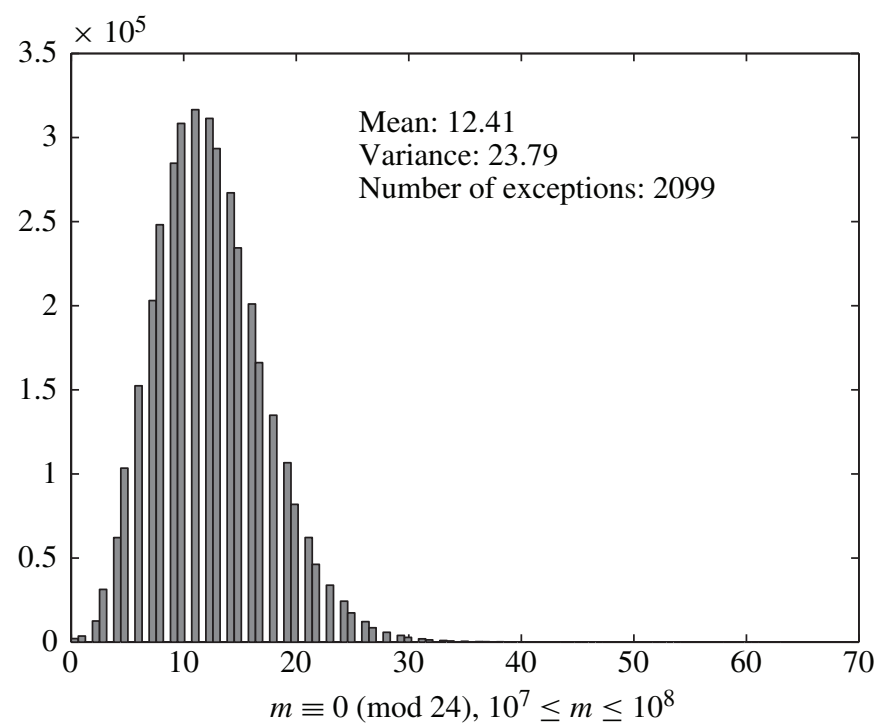

Figure 13.

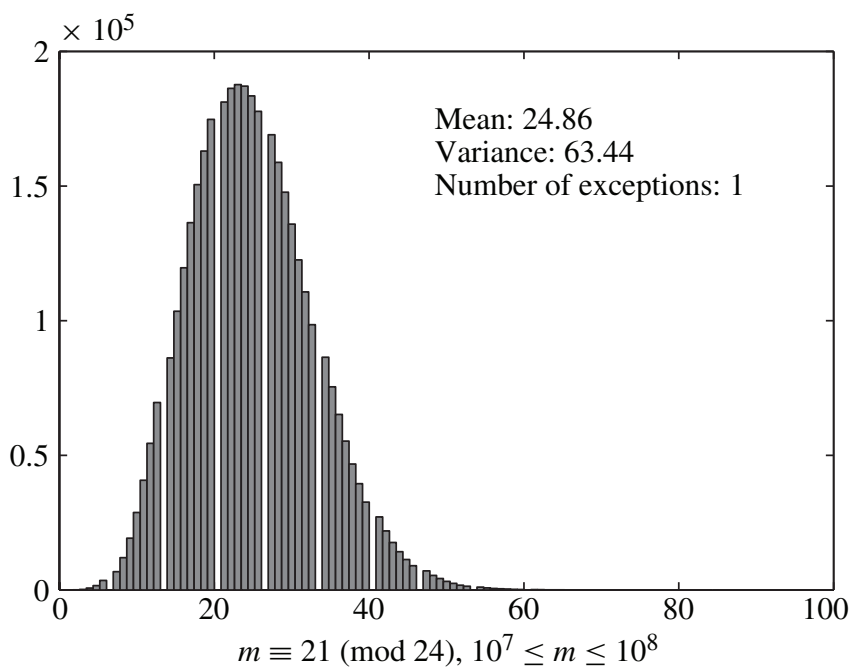

Figure 14.

global principle needs to be modified beyond the naive congruence obstructions, there being obstructions coming from the spin group [4, p. 250]. Given this, one should be cautious about a local-to-global conjecture in the context of the small group $A$, but my guess is, something like this is true, and to me this problem is a fundamental and attractive one.

One can ask which pairs of positive integers are curvatures of circles $C_{1}, C_{2}$ in $P_{0}$ (here and below the pairs $C_{1}, C_{2}$ are unordered). Again there are some congruence obstructions, but this time there cannot be a stable (i.e., except for finitely many exceptions) local-to-global principle. The reason is that such pairs of circles are too sparse: Let

$$
N_{P}^{(2)}(x)=\mid\left\{C_{1}, C_{2} \in P \mid C_{1} \text { is tangent to } C_{2} \text { and } a\left(C_{1}\right), a\left(C_{2}\right) \leq x\right\} \mid .
$$


In generating the packing, a circle placed at generation $n>1$ is tangent to exactly three circles from previous generations, and its radius is no bigger than any of these three. From this it follows that

$$
N_{P}^{(2)}(x)=3 N_{P}(x)-6,
$$

and this is too small to accommodate even infinitely many local congruence obstructions.

6. PRIMES. If you are drawn to primes, then on looking at Figure 6 you might have asked if there are infinitely many circles whose curvatures are prime. Are there infinitely many "twin primes," that is, pairs of tangent circles both of whose curvatures are prime? The pair near the middle with curvatures 157 and 397 is such a twin. If these sets are infinite then can one count them asymptotically; is there a "prime number theorem"?

Theorem [18]. In any primitive integral Apollonian packing there are infinitely many twin primes, and in particular infinitely many circles whose curvatures are prime. In fact, the set of points $x$ in an orbit $\mathcal{O}_{a}=a \cdot$ A of a primitive integral point a $\in$ $V^{\text {prim }}(\mathbb{Z})$ for which at least two of $x$ 's coordinates are prime is Zariski dense in $V$.

For congruence reasons (even-odd), $P_{0}$ contains no prime triples, that is, mutually tangent circles $C, C^{\prime}, C^{\prime \prime}$ all of whose curvatures are prime. The proof of the above theorem uses the arithmetic subgroups $B_{1}, B_{2}, B_{3}$, and $B_{4}$ of $A$ to place the problem in the ballpark of more standard problems concerning primes. Eventually the halfdimensional sieve [12] is what is used to produce primes.

We turn to counting these primes and twin primes. What makes this feasible is the affine linear sieve introduced recently in [2]. This sieve applies to orbits of groups such as the Apollonian group and it achieves in this context roughly what the sieves of Brun and Selberg do in the classical setting of the integers. Let

$$
\Pi_{P}(x)=\mid\{C \in P: a(C) \leq x, a(C) \text { prime }\} \mid
$$

and define the closely related weighted count

$$
\psi_{P}(x)=\sum_{\substack{C \in P \\ a(C) \text { prime } \\ a(C) \leq x}} \log a(C) .
$$

For twin primes set

$$
\Pi_{P}^{(2)}(x)=\mid\left\{C, C^{\prime} \in P \mid a(C), a\left(C^{\prime}\right) \leq x, a(C), a\left(C^{\prime}\right) \text { prime, } C \text { tangent to } C^{\prime}\right\} \mid
$$

and define the corresponding weighted count

$$
\psi_{P}^{(2)}(x)=\sum_{\begin{array}{c}
C, C^{\prime} \in P \\
a(C), a\left(C^{\prime}\right) \leq x \\
a(C), a\left(C^{\prime}\right) \text { prime } \\
C \text { tangent to } C^{\prime}
\end{array}} \log a(C) \log a\left(C^{\prime}\right) .
$$

The asymptotics of $\Pi_{P}(x)$ and $\psi_{P}(x)$ are related on summing by parts: $\Pi_{P}(x) \sim$ $\psi_{P}(x) / \log x$ as $x \rightarrow \infty$. For $\Pi_{P}^{(2)}(x)$ and $\psi_{P}^{(2)}(x)$ the relation is less clear $\left(\gamma_{1} \leq\right.$ 
$\Pi_{P}^{(2)}(x)(\log x)^{2} / \psi_{P}^{(2)}(x) \leq \gamma_{2}$ for $0<\gamma_{1}<\gamma_{2}<\infty$ constants) and it is more natural to consider the weighted sum.

Using the affine sieve and standard heuristics concerning the randomness of the Möbius function $\mu(n)$ and a nontrivial calculation, Fuchs and Sanden [9] formulate a precise "prime number conjecture":

Conjecture [9]. For any primitive integral packing $P$, as $x \rightarrow \infty$

$$
\frac{\psi_{P}(x)}{N_{P}(x)} \longrightarrow L\left(2, \chi_{4}\right)
$$

and

$$
\frac{\psi_{P}^{(2)}(x)}{N_{P}^{(2)}(x)} \longrightarrow \beta
$$

where the numbers $L\left(2, \chi_{4}\right)$ and $\beta$ are

$$
\begin{aligned}
L\left(2, \chi_{4}\right) & =\prod_{p \equiv 1(\bmod 4)}\left(1-p^{-2}\right)^{-1} \cdot \prod_{p \equiv 3(\bmod 4)}\left(1+p^{-2}\right)^{-1}=0.9159 \ldots, \\
\beta & =\frac{2}{3} \cdot \prod_{p \equiv 1(\bmod 4)}\left(1-p^{-2}\right)^{-2} \cdot \prod_{p \equiv 3(\bmod 4)}\left(1+p^{-2}\right)^{-2} \cdot\left(1-2 p(p-1)^{-2}\right) \\
& =0.460 \ldots
\end{aligned}
$$

These numbers come from a detailed examination of the set $\mathcal{O}_{a}(q)$ and certain algebraically defined subsets thereof, which eventually leads to the product of the local densities over primes. It is a pleasant and unexpected feature that the prime and twin prime constants above don't depend on the packing $P$. A numerical check of these conjectures for $P_{0}$ with $x$ up to $10^{8}$ is given in the graphs in Figures 15 and 16. The graph in Figure 15 is that of $\psi_{P}(x) / N_{P}(x)$ against $x$, and in Figure 16 of $\psi_{P}^{(2)}(x) /$ $N_{P}^{(2)}(x)$. The agreement with the conjecture is good.

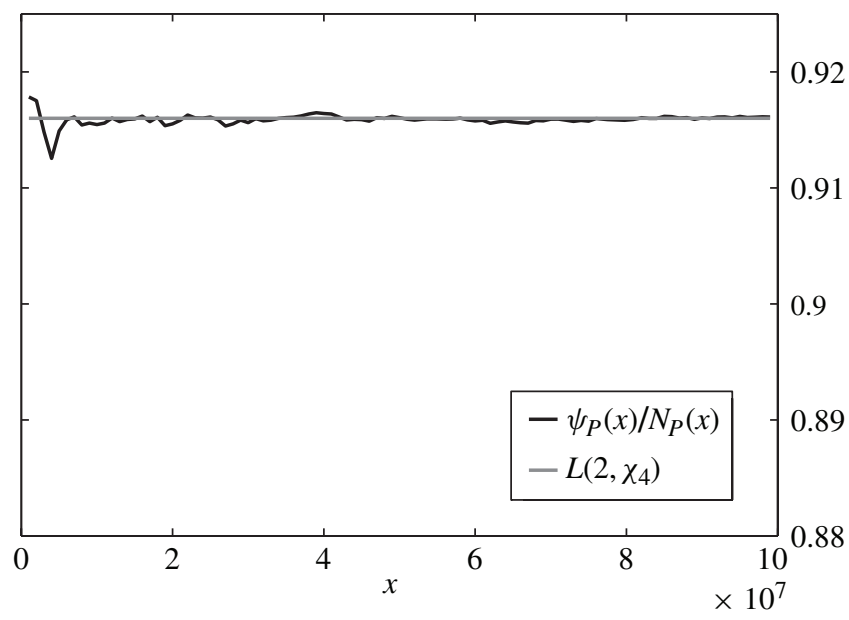

Figure 15. 


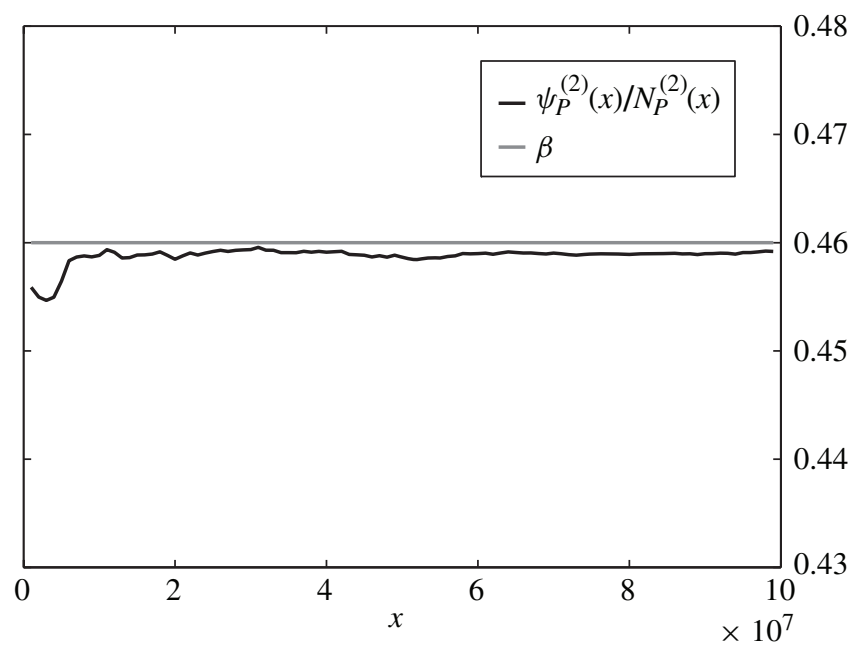

Figure 16.

As with the classical sieve the affine linear sieve can be used to prove upper bounds which are of the "true" order of magnitude.

Theorem [14]. Given a packing $P$ as above, there is constant $K$ depending on $P$ such that for $x \geq 2$,

$$
\Pi_{P}(x) \leq K \frac{N_{P}(x)}{\log x}
$$

and

$$
\Pi_{P}^{(2)}(x) \leq K \frac{N_{P}^{(2)}(x)}{(\log x)^{2}} .
$$

Note that from the refined asymptotics of [14] mentioned in Section 4, it follows that

$$
\sum_{C \in P} a(C)^{-\delta}=\infty
$$

According to the conjectured "prime number theorem" above we should have that

$$
\sum_{\substack{C \in P \\ a(C) \text { prime }}} a(C)^{-\delta}=\infty .
$$

On the other hand, the upper bound above for twins implies that

$$
\sum_{\substack{C, C^{\prime} \in P \\ C, C^{\prime} \text { twin primes }}}\left[\max \left(a(C), a\left(C^{\prime}\right)\right)\right]^{-\delta}<\infty .
$$

This is the analogue of Brun's theorem for the usual twin primes, which says that the sum of their reciprocals converges. 
There are many ingredients that go into the affine linear sieve and we end by mentioning one of them. For $q \geq 1$ the reduced orbit $\mathcal{O}_{a}(q)$ can be made into a 4-regular connected graph by joining $\xi$ in $\mathcal{O}_{a}(q)$ to $\xi S_{j}$ for $j=1,2,3,4$. The key property proved in [2] is that for $q$ square-free these graphs are an expander family as $q \longrightarrow \infty$ (see [18] and [11] for a definition and properties). This ensures that the random walk on $\mathcal{O}_{a}(q)$ gotten by moving with one of the $S_{j}$ at each step is rapidly uniformly mixing, and this is a critical ingredient in controlling remainder terms in the affine sieve.

ACKNOWLEDGMENTS. Thanks to Alex Kontorovich, Gerree Pecht, Katherine Sanden, and Ann Sarnak for providing the pictures, and the referee for pointing out some inaccuracies in an earlier version.

\section{REFERENCES}

1. J. Bourgain and E. Fuchs, On the density of integers appearing as curvatures in integer Apollonian circle packings, 2010 (preprint).

2. J. Bourgain, A. Gamburd, and P. Sarnak, Affine linear sieve, expanders and sum product, Invent. Math. 179 (2010) 559-644.

3. D. Boyd, The sequence of radii in an Apollonian packing, Math. Comp. 39 (1982) 249-254.

4. J. Cassels, Rational Quadratic Forms, Academic Press, London (1978).

5. J. Cogdell, Sums of three squares, J. Théor. Nombres Bordeaux 15 (2003) 33-44.

6. H. S. M. Coxeter, Loxodromic sequences of tangent spheres, Aequationes Math. 1 (1968) 104-121.

7. W. Duke and R. Schulze-Pillot, Representation of integers by positive ternary quadratic forms and equidistribution of lattice points on ellipsoids, Invent. Math. 99 (1990) 49-57.

8. E. Fuchs, Arithmetic properties of Apollonian circle packings, Ph.D. dissertation, Princeton University, Princeton, NJ, 2010.

9. E. Fuchs and K. Sanden, Prime number and local to global conjectures in Apollonian circle packings, 2009 (preprint).

10. R. L. Graham, J. C. Lagarias, C. L. Mallows, A. R. Wilks, and C.H. Yan, Apollonian circle packings: Number theory, J. Number Theory 100 (2003) 1-45.

11. S. Hoory, N. Linial, and A. Wigderson, Expander graphs and their applications, Bull. Amer. Math. Soc. (N.S) 43 (2006) 439-561.

12. H. Iwaniec, Primes represented by quadratic polynomials in two variables, Acta Arith. XXIV (1974) 435-459.

13. H. Iwanic and E. Kowalski, Analytic Number Theory, American Mathematical Society Colloquium Publications, vol. 53, American Mathematical Society, Providence, RI, 2004.

14. A. Kontorovich and $\mathrm{H}$. Oh, Apollonian packings and horospherical flows on hyperbolic 3-manifolds (2008), available at http://arxiv.org/abs/0811.2236v1.

15. É. LePage, Théorèmes limites pour les produits de matrices aléatoires, in Probability Measures on Groups: Proc. Sixth Conference, Oberwolfach, Germany, 1981, Lecture Notes in Mathematics, vol. 928, Springer, Berlin, 1982, 258-303.

16. C. Matthews, L. Vaserstein, and B. Weisfeiler, Congruence properties of Zariski dense groups, Proc. London Math. Soc. 45 (1984) 514-532.

17. C. McMullen, Hausdorff dimension and conformal dynamics III, computation of dimension, Amer. J. Math. 120 (1998) 691-721.

18. P. Sarnak, What is an expander? Notices Amer. Math. Soc. 51 (2004) 762-763.

19. Letter to Lagarias on integral Apollonian packings (2007), available at http://www. math. princeton.edu/sarnak/.

20. O. Wesler, An infinite packing theorem for spheres, Proc. Amer. Math. Soc. 11 (1960) 324-326.

PETER SARNAK is a professor at the Institute for Advanced Study in Princeton and at Princeton University. sarnak@math.princeton.edu 